BMJ Open

Diabetes

Research

\& Care

\section{Do glycemic marker levels vary by race? Differing results from a cross-sectional analysis of individuals with and without diagnosed diabetes}

To cite: Carson AP, Muntner P, Selvin E, et al. Do glycemic marker levels vary by race? Differing results from a cross-sectional analysis of individuals with and without diagnosed diabetes. BMJ Open Diabetes Research and Care 2016;4: e000213. doi:10.1136/ bmjdrc-2016-000213

- Additional material is available. To view please visit the journal online (http://dx. doi.org/10.1136/bmjdrc2016-000213)

Received 12 February 2016 Revised 5 April 2016 Accepted 9 May 2016

\section{ABSTRACT}

Objective: It is well known that $A 1 c$ varies by race. However, racial differences in other biomarkers of hyperglycemia are less well characterized. The objective of this study was to determine whether average levels of glycemic markers differ by race in adults with and without diagnosed diabetes, before and after accounting for postchallenge glucose.

Research design and methods: This crosssectional study included 2692 middle-aged men and women (5.5\% with diagnosed diabetes; $44 \%$ AfricanAmerican; and $56 \%$ white) from the Coronary Artery Risk Development in Young Adults Study (2005-2006) who had fasting glucose, 2-hour postchallenge glucose, A1c, glycated albumin, fructosamine, and 1,5-anhydroglucitol (1,5-AG) measured. Multiple linear regression was used to evaluate racial differences in mean levels of each glycemic marker stratified by the diabetes status and adjusted for sociodemographics, cardiovascular factors, and postchallenge glucose. Results: Among those with diagnosed diabetes, racial differences were not observed for any of the glycemic markers. In contrast, among those without diagnosed diabetes, African-Americans had higher mean levels than whites of $A 1 c$ ( $\beta=0.19 \%$ points; $95 \% \mathrm{Cl} 0.14$ to 0.24 ), glycated albumin ( $\beta=0.82 \%$ points; $95 \% \mathrm{Cl} 0.68$ to 0.97 ), fructosamine ( $\beta=8.68 \mu \mathrm{mol} / \mathrm{L} ; 95 \% \mathrm{Cl} 6.68$ to $10.68)$, and 2 -hour glucose $(\beta=3.50 \mathrm{mg} / \mathrm{dL} ; 95 \% \mathrm{Cl}$ 0.10 to 6.90 ) after multivariable adjustment, whereas there were no statistically significant racial difference in 1,5-AG. The racial differences observed for A1C, glycated albumin, and fructosamine persisted after further adjustment for fasting and 2-hour glucose and were of similar magnitude (SD units).

Conclusions: Racial differences in glycemic marker levels were evident among middle-aged adults without diagnosed diabetes even after adjustment for postchallenge glucose. Whether these racial differences in biomarkers of hyperglycemia affect the risk of complications warrants additional study.

\section{INTRODUCTION}

Current clinical practice recommendations identify fasting glucose, 2-hour postchallenge glucose, and hemoglobin A1c (A1c) as the

\section{Key messages}

- Hemoglobin A1c, glycated albumin, fructosamine, and 2-hour postchallenge glucose levels were higher for African-Americans than whites without diabetes.

- These racial differences were of similar magnitude and observed for multiple glycemic markers that reflect distinct aspects of glycemia.

- No racial differences in glycemia were observed for those with diagnosed diabetes.

standard glycemic markers for the assessment of type 2 diabetes and pre-diabetes status. ${ }^{1}$ However, racial differences in mean levels of these glycemic markers have been observed across the glycemic continuum. A1c has consistently been shown to be higher in African-Americans compared with whites among individuals with diabetes, ${ }^{2}{ }^{3}$ prediabetes, ${ }^{3}{ }^{4}$ and normal glycemia. ${ }^{3} 5$ In contrast to findings for A1c, mixed findings have been reported for fasting glucose and 2-hour glucose among individuals without diabetes, with studies reporting African-Americans having lower fasting glucose and 2-hour glucose levels than whites ${ }^{5}$ as well as no racial differences. ${ }^{6}$

Less commonly used glycemic markers, such as glycated albumin, fructosamine, and 1,5-anhydroglucitol (1,5-AG), reflect different aspects of glycemia and may provide insights into observed racial differences in measures of hyperglycemia. Glycated albumin and fructosamine are indicative of short-term glycemic control over 2-3 weeks based on glycation of serum proteins, ${ }^{7}$ whereas serum 1,5-AG concentrations reflect postprandial hyperglycemia in the setting of diabetes where lower levels result from glucose peaks above the renal threshold. ${ }^{8}$ In the Atherosclerosis Risk in Communities (ARIC) study, Alc, glycated albumin, and fructosamine levels were higher 
for African-Americans than whites among those with and without diabetes after multivariable adjustment. ${ }^{9}$ Furthermore, 1,5-AG and fasting glucose also varied by race among those without diabetes in the ARIC study, but assessments of 2-hour glucose were not available. ${ }^{9}$ An international study of 2094 individuals with diabetes reported that Africans residing in the USA had higher Alc than whites, yet more favorable 1,5-AG levels. ${ }^{10}$ Prior studies have not investigated standard glycemic markers, including 2-hour glucose, and emergent short-term and long-term glycemic markers, in a community-based cohort of individuals with and without diabetes. Thus, this study compared levels of fasting glucose, 2-hour glucose, A1c, glycated albumin, fructosamine, and 1,5-AG among African-American and white middle-aged adults with and without diagnosed diabetes from the Coronary Artery Risk Development in Young Adults (CARDIA) study.

\section{RESEARCH DESIGN AND METHODS}

\section{Study design and population}

CARDIA is a prospective cohort study designed to investigate trends and determinants of cardiovascular disease risk in the USA. A detailed overview of the study design, recruitment, and objectives has been previously published. ${ }^{11}$ Briefly, the baseline examination (1985-1986) included 5115 African-American and white men and women aged 18-30 years from Birmingham, Alabama; Chicago, Illinois; Minneapolis, Minnesota; and Oakland, California. Follow-up examinations were conducted at years 2 (1987-1988), 5 (1990-1991), 7 (1992-1993), 10 (1995-1996), 15 (2000-2001), 20 (2005-2006), and 25 (2010-2011) following the baseline examination. Participant retention rates for each of the follow-up examinations have been high, with $72 \%(\mathrm{n}=3549)$ of surviving participants completing the year 20 examination (baseline for this analysis). Centralized training and certification, standardized methods, and quality control measures were implemented to ensure high data quality for all examinations. ${ }^{12}$ The Institutional Review Board at each participating site approved all protocols and participants provided written informed consent at each study examination.

For the current cross-sectional analysis, only data collected at the year 20 examination (2005-2006) were used. We excluded 309 participants who were missing stored blood samples for the measurement of glycated albumin, fructosamine, or 1,5-AG; 398 missing A1c or fasting glucose; and 150 missing covariate data for a final sample size of 2692 participants. All analyses were stratified by diagnosed diabetes, defined as a self-report of a physician diagnosis of diabetes or use of insulin or oral hypoglycemic medications, resulting in a sample size of 147 participants with diagnosed diabetes and 2545 participants without diagnosed diabetes. Participants were ineligible for the oral glucose tolerance test to assess 2-hour glucose if they reported using steroids (except oral inhalers), insulin, or oral medications, or may have been pregnant. As a result, 2-hour glucose was not assessed for those with diagnosed diabetes and the sample size for the analysis of 2-hour glucose was 2164 for those without diagnosed diabetes.

\section{Glycemic markers}

Fasting glucose was measured using a hexokinase method coupled with glucose-6-phosphate dehydrogenase. After the initial venipuncture, participants who met eligibility criteria for the oral glucose tolerance test were asked to drink a $75 \mathrm{~g}$ glucose solution for the oral glucose tolerance test and had a second venipuncture 2 hours later to obtain 2-hour glucose measured using the hexokinase method. A1c was measured from a whole blood aliquot by ion-exchange high-performance liquid chromatography using a Tosoh G7 (Tosoh Bioscience, San Francisco, California, USA). ${ }^{13}$ Fasting glucose, 2-hour glucose, and A1c were analyzed at the University of Minnesota during the year 20 examination (20052006). The interassay coefficients of variation were $1.9 \%$ for fasting glucose, $3.5 \%$ for 2-hour glucose, and $1.3 \%$ for A1c.

Serum specimens from the year 20 examination were stored at $-70^{\circ} \mathrm{C}$ and used to analyze glycated albumin, fructosamine, and 1,5-AG in 2014 using a Roche COBAS 6000 chemistry analyzer (Roche Diagnostics Corporation, Indianapolis, Indiana, USA) at the University of Minnesota. Glycated albumin was measured using an enzymatic method coupled with a colorimetric method using an albumin-specific proteinase, ketoamine oxidase, and albumin assay reagent (Lucica GA-L, Asahi Kasei Pharma Corporation, Tokyo, Japan). ${ }^{14}$ Fructosamine was measured using a colorimetric method to detect total glycated serum proteins resulting from the reduction of nitro blue tetrazolium by ketoamines (Roche Diagnostics). ${ }^{15}$ 1,5-AG was measured using an enzymatic assay coupled with a colorimetric method to detect the oxidation of 1,5-AG by peroxidase (GlycoMark, Winston-Salem, North Carolina, USA).$^{16}{ }^{17}$ The interassay coefficients of variation were $2.9 \%$ for glycated albumin, $1.8 \%$ for fructosamine, and $3.6 \%$ for 1,5-AG. The glycated albumin assay demonstrated stable performance in samples stored over 20 years and high correlation with A1c. ${ }^{18}$

\section{Ascertainment of covariates}

Data on age, sex, race, education, and smoking status were collected using standardized questionnaires. Height and weight were measured by a certified study personnel and were used to calculate body mass index (BMI) in $\mathrm{kg} / \mathrm{m}^{2}$. After resting for $5 \mathrm{~min}$ in the seated position, blood pressure was measured three times at 1 min intervals using an appropriate sized cuff and a standard automated measurement monitor (Omron model HEM907XL), with the average of the second and third measurements used to determine systolic blood pressure (SBP). Total cholesterol was measured enzymatically, HDL-cholesterol was determined by precipitation 
with dextran sulfate-magnesium chloride, and LDL-cholesterol was estimated from the Friedewald equation. ${ }^{19}$ Medication use was ascertained by a questionnaire and a pill bottle review.

\section{Statistical analysis}

All analyses were stratified by the diagnosed diabetes status. Racial differences in participant characteristics were assessed using $\chi^{2}$, Fisher's exact, and t- tests as appropriate. Multiple regression was used to assess differences in mean levels of each glycemic marker for African-Americans compared with whites. Model 1 adjusted for age, sex, and education. Model 2 adjusted for model 1 variables plus study field center, smoking status, BMI, SBP, use of antihypertensive medications, total cholesterol, HDL-cholesterol, and use of lipid-lowering medications. Model 3 adjusted for model 2 variables plus 2-hour glucose (for outcomes other than 2-hour glucose) and fasting glucose (for outcomes other than fasting glucose) for those without diagnosed diabetes. Since the glycemic markers have different measurement units, an additional analysis used multiple regression to evaluate racial differences in standardized values (mean $=0$ and $\mathrm{SD}=1$ ) of each glycemic marker stratified by diabetes status. Sensitivity analyses were conducted for individuals with diagnosed diabetes to adjust for diabetes medication and for individuals without diagnosed diabetes who had normal glucose tolerance (2-hour glucose $<140 \mathrm{mg} / \mathrm{dL}$ ). All statistical analyses were conducted using SAS V.9.4 (SAS Institute, Cary, North Carolina, USA).

\section{RESULTS}

Participant characteristics are presented overall and stratified by diagnosed diabetes status in table 1 . Diagnosed diabetes was more common among African-Americans $(8.0 \%)$ than whites $(3.4 \%)$. Among those with diagnosed diabetes, participant characteristics were similar, except African-Americans were younger, more likely to be current smokers, and had higher HDL compared with whites. Among those without diagnosed diabetes, African-Americans had more adverse health characteristics than whites, including higher BMI, higher SBP, and a parental history of diabetes.

The correlations among glycemic markers are presented by diagnosed diabetes status in online supplementary table S1. Among participants with diagnosed diabetes, the glycemic markers were moderately to strongly correlated overall. Fasting glucose was positively correlated with Alc (0.76), glycated albumin (0.77), and fructosamine (0.78), and inversely correlated with 1,5-AG (-0.59). A1c exhibited a similar pattern with positive correlations with glycated albumin (0.84) and fructosamine $(0.80)$, and a slightly stronger inverse correlation with 1,5-AG (-0.76). Among the emerging glycemic markers, glycated albumin and fructosamine exhibited the strongest correlation (0.94).

Among participants without diagnosed diabetes, the correlations were mostly weak to moderate. Fasting glucose was positively correlated with Alc (0.37) and 2-hour glucose (0.31), but exhibited weak correlations with glycated albumin (0.04) or 1,5-AG (0.02). Two-hour glucose was positively correlated with Alc (0.26),

Table 1 Participant characteristics stratified by diagnosed diabetes status and race, the Coronary Artery Risk Development in Young Adults study 2005-2006

\begin{tabular}{|c|c|c|c|c|c|c|c|}
\hline \multirow[b]{2}{*}{ Characteristics $^{*}$} & \multirow[b]{2}{*}{$\begin{array}{l}\text { Overall } \\
(\mathrm{N}=2692)\end{array}$} & \multicolumn{3}{|c|}{$\begin{array}{l}\text { Diagnosed diabetes } \\
(\mathrm{N}=147)\end{array}$} & \multicolumn{3}{|c|}{$\begin{array}{l}\text { No diagnosed diabetes } \\
(\mathrm{N}=2545)\end{array}$} \\
\hline & & $\begin{array}{l}\text { African- } \\
\text { American } \\
(\mathrm{N}=96)\end{array}$ & $\begin{array}{l}\text { White } \\
(\mathrm{N}=51)\end{array}$ & p Value & $\begin{array}{l}\text { African- } \\
\text { American } \\
(\mathrm{N}=1100)\end{array}$ & $\begin{array}{l}\text { White } \\
(\mathrm{N}=1445)\end{array}$ & p Value \\
\hline Age, years & $45.3 \pm 3.6$ & $44.9 \pm 3.8$ & $46.6 \pm 3.2$ & 0.008 & $44.7 \pm 3.8$ & $45.7 \pm 3.3$ & $<0.001$ \\
\hline$\%$ Women & 54.5 & 59.4 & 33.3 & 0.003 & 58.7 & 51.7 & $<0.001$ \\
\hline$\%$ High school education or less & 24.6 & 33.3 & 33.3 & 0.205 & 35.2 & 15.6 & $<0.001$ \\
\hline$\%$ Current smoker & 19.6 & 21.9 & 15.7 & 0.040 & 25.1 & 15.4 & $<0.001$ \\
\hline Body mass index, $\mathrm{kg} / \mathrm{m}^{2}$ & $29.3 \pm 6.7$ & $35.3 \pm 8.0$ & $33.6 \pm 7.5$ & 0.212 & $30.7 \pm 7.1$ & $27.7 \pm 5.7$ & $<0.001$ \\
\hline Systolic blood pressure, $\mathrm{mm} \mathrm{Hg}$ & $116.5 \pm 15.1$ & $121.9 \pm 18.0$ & $118.2 \pm 17.6$ & 0.239 & $120.2 \pm 16.1$ & $113.2 \pm 13.1$ & $<0.001$ \\
\hline$\%$ Use antihypertensive medications & 17.2 & 50.0 & 47.1 & 0.734 & 23.1 & 9.4 & $<0.001$ \\
\hline Total cholesterol, mg/dL & $186.2 \pm 34.9$ & $179.3 \pm 38.1$ & $182.9 \pm 45.0$ & 0.605 & $184.2 \pm 35.5$ & $188.3 \pm 33.6$ & 0.003 \\
\hline HDL cholesterol, mg/dL & $54.1 \pm 16.7$ & $49.6 \pm 15.1$ & $42.5 \pm 13.9$ & 0.006 & $54.8 \pm 16.3$ & $54.2 \pm 17.0$ & 0.349 \\
\hline LDL cholesterol, mg/dL & $110.5 \pm 32.1$ & $106.8 \pm 34.1$ & $104.4 \pm 38.2$ & 0.702 & $110.6 \pm 33.7$ & $111.0 \pm 30.5$ & 0.762 \\
\hline$\%$ Use lipid-lowering medications & 9.2 & 35.4 & 43.1 & 0.359 & 5.8 & 8.8 & 0.005 \\
\hline$\%$ Parental history of diabetes & 32.8 & 51.0 & 47.1 & 0.646 & 38.7 & 26.6 & $<0.001$ \\
\hline$\% A 1 c \geq 7 \%(\geq 53 \mathrm{mmol} / \mathrm{mol})$ & 3.9 & 50.0 & 35.3 & 0.088 & 2.5 & 0.8 & $<0.001$ \\
\hline \multicolumn{8}{|l|}{ Diabetes medication type } \\
\hline$\%$ Insulin only & - & 1.0 & 9.8 & 0.041 & - & - & \\
\hline$\%$ Insulin and oral agents & - & 8.3 & 3.9 & & - & - & \\
\hline$\%$ Oral hypoglycemic agents only & - & 57.3 & 52.9 & & - & - & \\
\hline
\end{tabular}


whereas it was weakly correlated with glycated albumin (0.06), fructosamine (0.05), and 1,5-AG (-0.08). Additionally, scatterplots and histograms for the distribution of the glycemic markers are presented in online supplementary tables S2-S6 for those with diagnosed diabetes and online supplementary tables S7-S12 for those without diagnosed diabetes.

The crude mean and median glycemic marker levels by diagnosed diabetes status and race are presented in table 2. For individuals with diagnosed diabetes, there were no statistically significant racial differences in average levels for any of the glycemic markers. In contrast, racial differences were observed for several glycemic markers among individuals without diagnosed diabetes. African-Americans had higher Alc, higher glycated albumin, higher fructosamine, and higher 2-hour glucose, but no statistically significant differences were noted for fasting glucose or 1,5-AG.

For multiple regression analyses, residual plots were similar using non-transformed and transformed outcomes, so analyses are presented for non-transformed markers. The multiple regression models are presented in table 3. Among individuals with diagnosed diabetes, there were no statistically significant differences in glycemic marker levels for African-Americans compared with whites in minimally adjusted or fully adjusted models. In sensitivity analyses with additional adjustment for diabetes medication, the results did not change (data not shown). However, racial differences were apparent for some glycemic markers among individuals without diagnosed diabetes. African-Americans without diagnosed diabetes had higher A1c $(\beta=0.19 \%$ points, $95 \%$ CI 0.14 to 0.24$)$, glycated albumin ( $\beta=0.82 \%$ points, $95 \%$ CI 0.68 to 0.97$)$, and fructosamine $(\beta=8.68 \mu \mathrm{mol} / \mathrm{L}$, $95 \%$ CI 6.68 to 10.68 ) than whites without diagnosed diabetes. With further adjustment for 2-hour glucose and fasting glucose, racial differences in $\mathrm{A} 1 \mathrm{c}(\beta=0.19 \%$ points, $95 \%$ CI 0.15 to 0.23 ), glycated albumin ( $\beta=0.79 \%$ points, $95 \%$ CI 0.68 to 0.91 ), and fructosamine $(\beta=8.29 \mu \mathrm{mol} / \mathrm{L}, \quad 95 \%$ CI 6.60 to 9.99$)$ persisted. African-Americans without diagnosed diabetes also had higher 2-hour glucose and lower fasting glucose after multivariable adjustment, whereas no significant racial differences were observed for 1,5-AG. In a sensitivity analysis of those without diagnosed diabetes and who had normal glucose tolerance (2-hour glucose $<140 \mathrm{mg} / \mathrm{dL}$ ), African-Americans had lower FG $(\beta=-1.60 \mathrm{mg} / \mathrm{dL}, 95 \%$ CI -2.47 to -0.72 ), higher A1c ( $\beta=0.16 \%$ points, $95 \%$ CI 0.12 to 0.20$)$, higher glycated albumin $(\beta=0.70 \%$ points, $95 \%$ CI 0.59 to 0.80 ), and higher fructosamine $(\beta=6.60 \mu \mathrm{mol} / \mathrm{L}, 95 \%$ CI 4.97 to 8.22$)$ than whites after multivariable adjustment, whereas no racial differences were observed in 2-hour glucose and 1,5-AG.

The analysis of standardized glycemic marker levels is presented in figure 1. The SDs were $71.77 \mathrm{mg} / \mathrm{dL}$ for fasting glucose, $2.11 \%(23.1 \mathrm{mmol} / \mathrm{mol})$ for A1c, $6.71 \%$ for glycated albumin, $84.22 \mu \mathrm{mol} / \mathrm{L}$ for fructosamine, and $7.90 \mu \mathrm{g} / \mathrm{mL}$ for $1,5-\mathrm{AG}$ for those with diagnosed diabetes. Among participants without diagnosed diabetes, the SDs were $15.15 \mathrm{mg} / \mathrm{dL}$ for fasting glucose, $0.59 \%$ $(6.4 \mathrm{mmol} / \mathrm{mol})$ for A1c, $1.69 \%$ for glycated albumin, $23.96 \mu \mathrm{mol} / \mathrm{L}$ for fructosamine, $6.14 \mu \mathrm{g} / \mathrm{mL}$ for $1,5-\mathrm{AG}$,

Table 2 Comparison of crude glycemic marker levels by diagnosed diabetes status and race, the Coronary Artery Risk Development in Young Adults study 2005-2006

\begin{tabular}{|c|c|c|c|c|c|c|}
\hline \multirow[b]{2}{*}{ Glycemic marker } & \multicolumn{3}{|l|}{ Diagnosed diabetes } & \multicolumn{3}{|c|}{ No diagnosed diabetes } \\
\hline & $\begin{array}{l}\text { African-American } \\
(\mathrm{N}=96)\end{array}$ & $\begin{array}{l}\text { White } \\
(\mathrm{N}=51)\end{array}$ & p Value* & $\begin{array}{l}\text { African-American } \\
(\mathrm{N}=1100)\end{array}$ & $\begin{array}{l}\text { White } \\
(\mathrm{N}=1445)\end{array}$ & p Value \\
\hline \multicolumn{7}{|c|}{ Fasting glucose (mg/dL) } \\
\hline Mean \pm SD & $151.2 \pm 70.2$ & $155.6 \pm 75.3$ & \multirow[t]{2}{*}{0.724} & $96.5 \pm 17.7$ & $95.8 \pm 12.9$ & \multirow[t]{2}{*}{0.274} \\
\hline Median (IQR) & $130.2(104.8-175.9)$ & $132.6(103.4-181.3)$ & & $94.6(87.8-101.4)$ & $94.6(89.8-100.5)$ & \\
\hline \multicolumn{7}{|l|}{ A1c $(\%)$} \\
\hline Mean \pm SD & $7.5 \pm 2.0$ & $7.3 \pm 2.4$ & \multirow[t]{2}{*}{0.587} & $5.6 \pm 0.7$ & $5.3 \pm 0.5$ & \multirow[t]{2}{*}{$<0.001$} \\
\hline Median (IQR) & $7.0(6.0-8.6)$ & $6.4(5.9-7.9)$ & & $5.5(5.2-5.8)$ & $5.3(5.1-5.5)$ & \\
\hline \multicolumn{7}{|c|}{ Glycated albumin (\%) } \\
\hline Mean \pm SD & $18.4 \pm 6.7$ & $17.4 \pm 6.7$ & \multirow[t]{2}{*}{0.390} & $13.2 \pm 2.0$ & $12.6 \pm 1.4$ & \multirow[t]{2}{*}{$<0.001$} \\
\hline Median (IQR) & $16.3(14.0-22.0)$ & $14.2(12.9-19.6)$ & & $13.0(12.2-13.8)$ & $12.5(11.7-13.3)$ & \\
\hline \multicolumn{7}{|c|}{ Fructosamine $(\mu \mathrm{mol} / \mathrm{L})$} \\
\hline Mean \pm SD & $293.3 \pm 83.6$ & $285.4 \pm 86.0$ & \multirow[t]{2}{*}{0.586} & $229.8 \pm 27.6$ & $225.7 \pm 20.6$ & \multirow[t]{2}{*}{$<0.001$} \\
\hline Median (IQR) & $270.0(235.5-339.0)$ & $256.0(234.0-301.0)$ & & $228.0(214.0-241.0)$ & 225 (213-237) & \\
\hline \multicolumn{7}{|c|}{ 1,5-Anhydroglucitol ( $\mu \mathrm{g} / \mathrm{mL})$} \\
\hline Mean \pm SD & $10.7 \pm 8.1$ & $10.5 \pm 7.6$ & \multirow[t]{2}{*}{0.914} & $18.8 \pm 6.3$ & $18.8 \pm 6.0$ & \multirow[t]{2}{*}{0.979} \\
\hline Median (IQR) & $9.2(3.2-17.9)$ & $10.8(3.6-17.4)$ & & $18.8(15.0-22.8)$ & $18.8(15.0-22.6)$ & \\
\hline \multicolumn{7}{|c|}{ 2-hour postchallenge glucose† (mg/dL) } \\
\hline Mean $\pm S D$ & - & - & \multirow[t]{2}{*}{-} & $111.3 \pm 40.7$ & $102.7 \pm 33.2$ & \multirow[t]{2}{*}{$<0.001$} \\
\hline Median (IQR) & - & - & & $103.4(87.8-123.8)$ & $97.5(82.0-115.1)$ & \\
\hline
\end{tabular}


Table 3 Adjusted $\beta$ coefficients for glycemic marker mean levels comparing African-Americans with whites among individuals with and without diagnosed diabetes, the Coronary Artery Risk Development in Young Adults study 2005-2006

\begin{tabular}{|c|c|c|c|c|c|c|}
\hline \multirow[b]{2}{*}{ Glycemic marker } & \multicolumn{3}{|l|}{ Diagnosed diabetes } & \multicolumn{3}{|l|}{ No diagnosed diabetes } \\
\hline & $\begin{array}{l}\text { African-American } \\
\text { versus White }\end{array}$ & p Value & Model R ${ }^{2}$ & $\begin{array}{l}\text { African-American } \\
\text { versus White }\end{array}$ & p Value & Model $\mathbf{R}^{2}$ \\
\hline \multicolumn{7}{|c|}{ Fasting glucose (mg/dL) } \\
\hline Model 1 & $-3.79(-29.89$ to 22.31$)$ & 0.775 & 0.04 & $1.05(-0.22$ to 2.31$)$ & 0.106 & 0.03 \\
\hline Model 2 & $-3.13(-30.71$ to 24.44$)$ & 0.823 & 0.19 & $-0.35(-1.62$ to 0.93$)$ & 0.594 & 0.14 \\
\hline Model 3 & - & - & - & $-1.06(-2.04$ to -0.08$)$ & 0.033 & 0.39 \\
\hline \multicolumn{7}{|l|}{$\mathrm{A} 1 \mathrm{c}(\%)$} \\
\hline Model 1 & $0.24(-0.54$ to 1.01$)$ & 0.548 & 0.02 & 0.24 (0.19 to 0.29$)$ & $<0.001$ & 0.06 \\
\hline Model 2 & $0.17(-0.64$ to 0.97$)$ & 0.684 & 0.20 & $0.19(0.14$ to 0.24$)$ & $<0.001$ & 0.15 \\
\hline Model 3 & - & - & - & $0.19(0.15$ to 0.23$)$ & $<0.001$ & 0.45 \\
\hline \multicolumn{7}{|c|}{ Glycated albumin (\%) } \\
\hline Model 1 & $0.82(-1.65$ to 3.29$)$ & 0.512 & 0.02 & $0.74(0.60$ to 0.88$)$ & $<0.001$ & 0.05 \\
\hline Model 2 & $0.60(-1.95$ to 3.15$)$ & 0.642 & 0.20 & 0.82 (0.68 to 0.97$)$ & $<0.001$ & 0.09 \\
\hline Model 3 & - & - & - & 0.79 (0.68 to 0.91$)$ & $<0.001$ & 0.38 \\
\hline \multicolumn{7}{|c|}{ Fructosamine ( $\mu \mathrm{mol} / \mathrm{L})$} \\
\hline Model 1 & $8.47(-22.31$ to 39.24$)$ & 0.587 & 0.03 & 6.60 (4.61 to 8.60$)$ & $<0.001$ & 0.03 \\
\hline Model 2 & $11.85(-19.73$ to 43.43$)$ & 0.459 & 0.22 & 8.68 (6.68 to 10.68$)$ & $<0.001$ & 0.16 \\
\hline Model 3 & - & - & - & 8.29 (6.60 to 9.99$)$ & $<0.001$ & 0.40 \\
\hline \multicolumn{7}{|c|}{ 1,5-Anhydroglucitol ( $\mu \mathrm{g} / \mathrm{mL})$} \\
\hline Model 1 & $0.17(-2.73$ to 3.06$)$ & 0.910 & 0.03 & $-0.22(-0.71$ to 0.26$)$ & 0.368 & 0.11 \\
\hline Model 2 & $0.15(-2.98$ to 3.27$)$ & 0.927 & 0.14 & $-0.49(-1.01$ to 0.02$)$ & 0.062 & 0.14 \\
\hline Model 3 & - & - & - & $-0.36(-0.91$ to 0.18$)$ & 0.189 & 0.17 \\
\hline \multicolumn{7}{|c|}{ 2-hour postchallenge glucose* (mg/dL) } \\
\hline Model 1 & - & - & - & $7.80(4.45$ to 11.14$)$ & $<0.001$ & 0.02 \\
\hline Model 2 & - & - & - & $3.50(0.10$ to 6.90$)$ & 0.044 & 0.13 \\
\hline Model 3 & - & - & - & 4.18 (1.29 to 7.07$)$ & 0.005 & 0.37 \\
\hline \multicolumn{7}{|c|}{$\begin{array}{l}\text { Model } 1 \text { adjusted for age, sex, and education. Model } 2 \text { adjusted for Model } 1 \text { variables plus study field center, smoking status, body mass } \\
\text { index, systolic blood pressure, use of antihypertensive medications, total cholesterol, HDL cholesterol, and use of lipid-lowering medications. } \\
\text { Model } 3 \text { adjusted for model } 2 \text { variables plus } 2 \text {-hour glucose (for markers other than } 2 \text {-hour glucose) and fasting glucose (for markers other } \\
\text { than fasting glucose). }\end{array}$} \\
\hline
\end{tabular}

and $36.81 \mathrm{mg} / \mathrm{dL}$ for 2-hour glucose. After adjustment for age, sex, education, study field center, smoking status, BMI, SBP, use of antihypertensive medications, total cholesterol, HDL cholesterol, and use of lipid-lowering medications, no racial differences in any standardized glycemic markers were observed for individuals with diagnosed diabetes. In contrast, among individuals without diagnosed diabetes, African-Americans had lower fasting glucose $(-0.07$ SDs, $95 \%$ CI -0.13 to -0.01$)$, higher Alc (0.33 SDs, $95 \%$ CI 0.26 to 0.39 ), higher glycated albumin (0.47 SDs, $95 \%$ CI 0.40 to 0.54 ), higher fructosamine (0.35 SDs, $95 \%$ CI 0.28 to 0.42 ), and higher 2-hour glucose $(0.11$ SDs, $95 \%$ CI 0.04 to 0.19$)$ compared with whites after adjustment for demographics, cardiovascular risk factors, fasting glucose (for outcomes other than fasting glucose), and 2-hour glucose (for outcomes other than 2-hour glucose). No statistically significant racial differences were observed for standardized 1,5-AG.

\section{CONCLUSIONS}

In this cross-sectional analysis of multiple glycemic markers, no racial differences in mean glycemic marker levels were observed among participants with diagnosed diabetes. However, among those without diagnosed diabetes, mean A1c, glycated albumin, fructosamine, and 2-hour postchallenge glucose levels were higher for African-Americans than whites after adjustment for sociodemographic and cardiovascular factors. The findings were similar after further adjustment for fasting and 2-hour glucose, suggesting that postprandial hyperglycemia does not fully explain the observed racial differences in glycemic markers. A1c represents glycemic levels over 2-3 months and may be affected by red blood cell turnover, whereas glycated albumin and fructosamine represent glycemic levels over 2-3 weeks and may be affected by serum protein metabolism. ${ }^{720}$ Although these markers reflect glycemic levels over different time periods and may potentially be affected by different non-glycemic factors, the findings from this study show that African-Americans without diagnosed diabetes have higher levels of both short-term and long-term glycemic markers compared with whites without diagnosed diabetes irrespective of fasting and 2-hour glucose levels.

Previous studies of individuals with diabetes have reported racial and ethnic differences in glycemic marker levels. In the National Health and Nutrition Examination Survey 1999-2004, African-Americans with 
A

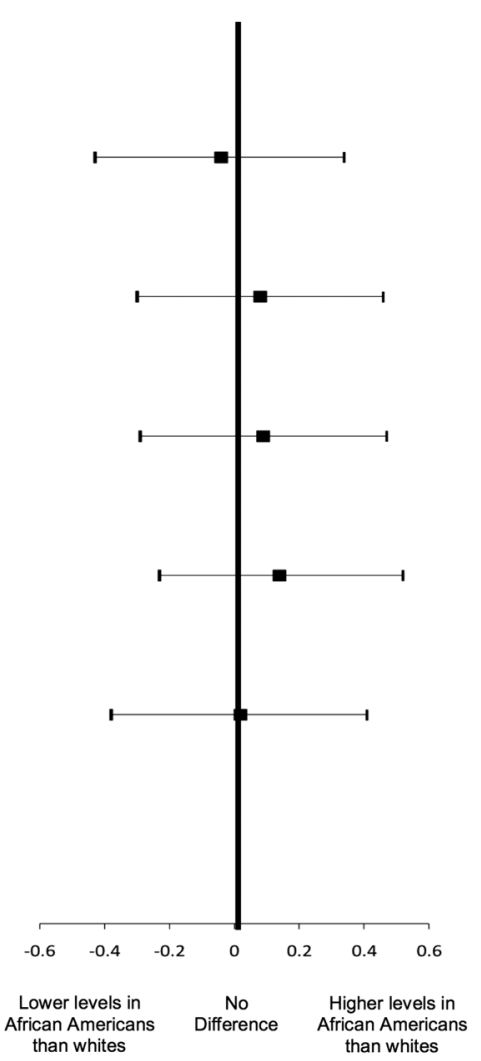

Fasting glucose

A1c

Glycated albumin

Fructosamine

1,5-anhydroglucitol

2-hour glucose
B

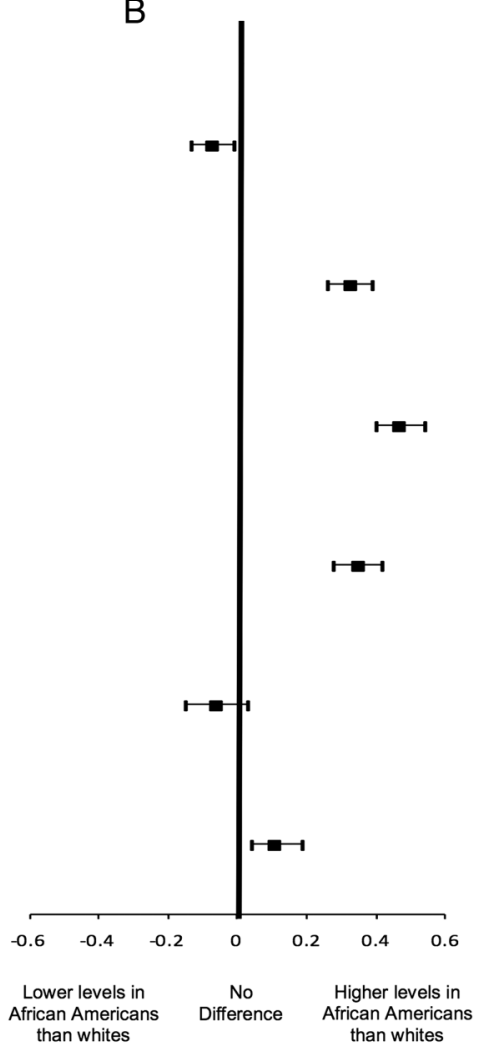

*Models adjusted for age, sex, education, study field center, smoking status, body mass index, systolic blood pressure, use of antihypertensive medications, total cholesterol, HDL cholesterol, use of lipidlowering medications, fasting glucose (without diagnosed diabetes only; except fasting glucose outcome) and 2-hour glucose (without diagnosed diabetes only; except 2-hour glucose outcome).

Figure 1 Adjusted ${ }^{*}$ standardized $\beta$ coefficients and $95 \%$ Cls for glycemic marker levels comparing African-Americans with whites among individuals with (A) and without (B) diagnosed diabetes, the Coronary Artery Risk Development in Young Adults study 2005-2006.

diagnosed diabetes had higher A1c levels than whites. ${ }^{21}$ Additionally, a meta-analysis of 11 studies reported that African-Americans with diabetes had $0.65 \%$ points higher Alc than whites with diabetes. ${ }^{2}$ In an international study of 2094 adults with type 2 diabetes, A1c was also $0.30 \%$ points higher for persons of African descent (majority lived in the USA) compared with whites, although paradoxically, persons of African descent had more favorable 1,5-AG levels than whites. ${ }^{10}$ This finding persisted after adjustment for multiple factors including diabetes duration, country of origin, and self-monitored plasma glucose level, but did not take into account differences in medication type. In the ARIC Study, African-Americans with diagnosed diabetes had higher A1c, glycated albumin, and fructosamine than whites with diagnosed diabetes after adjustment for sociodemographics and cardiovascular risk factors, while differences in fasting glucose and 1,5-AG were not statistically significant. ${ }^{9}$ In contrast, no racial differences were observed for any of the glycemic markers among those with diagnosed diabetes in the current study. Several factors may have contributed to the different findings observed in the current study including the low prevalence of diabetes in our study, younger population, and the similar cardiovascular health profile observed for African-Americans and whites with diabetes.

Among individuals without diagnosed diabetes, racial and ethnic differences in glycemic markers have also been demonstrated. In the Diabetes Prevention Program, A1c was higher for African-Americans with impaired glucose tolerance than whites with impaired glucose tolerance. ${ }^{4}$ Similarly, African-Americans had higher A1c than whites in another study that investigated individuals with impaired glucose tolerance and normal glycemia separately. ${ }^{3}$ Another study of normal glycemic adults with a parental history of type 2 diabetes also reported that African-Americans had higher A1c levels than whites. ${ }^{5}$ Prior studies have consistently reported racial differences in Alc, but few studies have investigated emerging shortterm glycemic markers among individuals without diabetes. In the ARIC Study, African-Americans without diabetes had higher levels of fasting glucose, Alc, glycated albumin, fructosamine, and lower 1,5-AG compared with whites, and these racial differences remained statistically significant after multivariable adjustment. ${ }^{9}$ In the current study, African-Americans without diabetes had higher Alc, glycated albumin, fructosamine, and 2-hour glucose levels than whites, while 1,5-AG levels were similar after 
multivariable adjustment. The magnitude of the differences was similar for A1c, glycated albumin, and fructosamine and persisted after additional adjustment for fasting glucose and 2-hour glucose, which have not been previously reported. A1c, glycated albumin, and fructosamine are affected by different factors (ie, red blood cell lifespan for A1c and serum protein metabolism for fructosamine and glycated albumin), so the underlying causes of the observed racial differences in these shortand long-term glycemic markers are unclear.

Among individuals with diagnosed diabetes, all of the glycemic markers were strongly correlated in the current study. The magnitude of the Spearman's correlations (0.59-0.84) for A1c and fasting glucose with glycated albumin, fructosamine, and 1,5-AG was similar in the current study to those reported in the full population of participants from the ARIC Study ${ }^{22}$ but stronger than those previously reported for a subsample of older participants. ${ }^{23}$ Among individuals without diagnosed diabetes, the correlations were moderate to weak. Alc, fasting glucose, and 2-hour glucose exhibited moderate correlations with each other $(0.26-0.37)$ and very weak or no correlations with glycated albumin, fructosamine, and 1,5-AG. Similarly, moderate to weak correlations were observed in the ARIC Study among individuals without diabetes for A1c and fasting glucose with glycated albumin, fructosamine, and 1,5-AG. ${ }^{23}$

The implications of these racial differences in glycemic markers among individuals without diagnosed diabetes are not clear. Elevated fasting glucose, 2-hour glucose, and A1c have been associated with macrovascular disease among individuals without diabetes. ${ }^{24-26}$ Findings on the association of emerging glycemic markers with microvascular and macrovascular outcomes among individuals without diagnosed diabetes are limited. Prior studies have reported that adverse levels of fructosamine, glycated albumin, and 1,5-AG were associated with albuminuria and retinopathy ${ }^{22} 28$ and incident diabetes. ${ }^{28-30}$ Additionally, glycated albumin, fructosamine, and 1,5-AG have been associated with an increased risk of coronary heart disease, ischemic stroke, heart failure, and all-cause mortality. ${ }^{31-33}$ Despite racial differences in glycemic marker levels, prior studies have not reported differences in the association of glycemic markers with cardiovascular outcomes and mortality. ${ }^{26}$ Further research is needed to evaluate the association of these glycemic markers with vascular outcomes in other populations and determine their utility for identifying individuals at high risk for developing diabetes and vascular outcomes.

This study has several potential limitations. First, fasting glucose, 2-hour glucose, and A1c were measured at the year 20 examination (2005-2006) while glycated albumin, fructosamine, and 1,5-AG were measured in 2014 using stored specimens collected at the year 20 examination. Prior studies have shown that stored specimens are stable for the assessment of glycated albumin ${ }^{18}$ and our central laboratory reported very good coefficients of variation for all of the assays (1.3-3.6\%). Second, the retention of CARDIA participants was high at the year 20 examination (72\%), but participants who did not attend the examination were excluded from this analysis and were more likely to be women, younger age, and African-American when compared with those included in this analysis. Third, a single measure of each glycemic marker was available for this analysis. While the different glycemic markers reflect varying time points across the glycemic spectrum, multiple assessments of these markers were not available. Finally, A1c levels may be altered by conditions that affect red blood cell turnover such as anemia, and glycated albumin and fructosamine levels may be altered by conditions that affect serum albumin metabolism, such as hyperthyroidism. In our study, these conditions were assessed by self-report and not objective measurements. This study also has numerous strengths, including the use of a wellestablished biracial cohort of middle-age adults from four communities in the USA, a central laboratory with excellent quality control to conduct all specimen analyses, and a comprehensive assessment of glycemic markers including 2-hour glucose.

In conclusion, racial differences in glycemic markers were evident for individuals without diagnosed diabetes but not for those with diagnosed diabetes in this community-based cohort of middle-aged adults. These findings were consistent and of similar magnitude across glycemic markers for those without diagnosed diabetes. The clinical implications, if any, of the observed racial differences in glycemic marker levels among individuals without diagnosed diabetes are not clear. Future studies should investigate the association of standard and emerging glycemic markers with microvascular and macrovascular outcomes to determine which glycemic markers best identify high-risk individuals and whether the predictive ability of these markers differ by race.

\section{Author affiliations}

${ }^{1}$ Department of Epidemiology, University of Alabama at Birmingham School of Public Health, Birmingham, Alabama, USA

${ }^{2}$ Department of Epidemiology, Johns Hopkins University Bloomberg School of Public Health, Baltimore, Maryland, USA

${ }^{3}$ Department of Preventive Medicine, Northwestern University Feinberg School of Medicine, Chicago, Illinois, USA

${ }^{4}$ Division of Preventive Medicine, University of Alabama at Birmingham School of Medicine, Birmingham, Alabama, USA

${ }^{5}$ Department of Laboratory Medicine and Pathology, University of Minnesota Medical School, Minneapolis, Minnesota, USA

${ }^{6}$ Department of Nutrition Sciences, University of Alabama at Birmingham School of Health Professions, Birmingham, Alabama, USA

${ }^{7}$ Birmingham VA Medical Center, Birmingham, Alabama, USA

Acknowledgments The authors thank the other investigators, the staff, and the participants of the CARDIA study for their contributions.

Contributors APC secured funding, conceptualized the study, designed the analysis, interpreted the data, and drafted the manuscript. PM, ES, MRC, and WTG interpreted the data and critically revised the manuscript for important intellectual content. XL performed the statistical analysis, interpreted the data, and critically revised the manuscript for important intellectual content. MDG 
and CEL secured funding, interpreted the data, and critically revised the manuscript for important intellectual content. APC is the guarantor of this work and, as such, had full access to all the data in the study and takes responsibility for the integrity of the data and the accuracy of the data analysis.

Funding The Coronary Artery Risk Development in Young Adults (CARDIA) study is supported by contracts HHSN268201300025C

HHSN268201300026C, HHSN268201300027C, HHSN268201300028C HHSN268201300029C, and HHSN268200900041C from the National Heart, Lung, and Blood Institute (NHLBI), the Intramural Research Program of the National Institute on Aging (NIA), and an intra-agency agreement between NIA and NHLBI (AG0005). Additional support provided by K01 DK09528 (PI: APC), R01 HL053560 (PI: MDG), the UAB Center for Clinical and Translational Science (UL1TR000165), and the UAB Diabetes Research Center (P30 DK079626). The assays for glycated albumin were provided in kind by Asahi Kasei Corporation (Tokyo, Japan). The assays for 1,5-AG were provided at a reduced research cost by GlycoMark (New York, New York, USA).

Competing interests Duality of interest: WTG is a consultant for AstraZeneca, Vivus, Liposcience, Daiichi-Sankyo, Janssen, Eisai, Takeda, Boehringer Ingelheim, and Novo Nordisk, is a shareholder with ISIS, Novartis, Bristol-Myers Squibb, Pfizer, Merck, and Lilly, and has received research grants from Merck, Weight Watchers, Sanofi, Eisai, AstraZeneca, Pfizer, Novo Nordisk, and Glaxo.

Ethics approval University of Alabama at Birmingham Institutional Review Board.

Provenance and peer review Not commissioned; externally peer reviewed.

Data sharing statement No additional data are available.

Open Access This is an Open Access article distributed in accordance with the Creative Commons Attribution Non Commercial (CC BY-NC 4.0) license, which permits others to distribute, remix, adapt, build upon this work noncommercially, and license their derivative works on different terms, provided the original work is properly cited and the use is non-commercial. See: http:// creativecommons.org/licenses/by-nc/4.0/

\section{REFERENCES}

1. American Diabetes Association. Classification and diagnosis of diabetes. Diabetes Care 2015;38:S8-16.

2. Kirk JK, D'Agostino RB Jr, Bell RA, et al. Disparities in HbA1c levels between African-American and non-Hispanic white adults with diabetes: a meta-analysis. Diabetes Care 2006;29:2130-6.

3. Ziemer DC, Kolm P, Weintraub WS, et al. Glucose-independent, black-white differences in hemoglobin A1c levels: a cross-sectional analysis of 2 studies. Ann Intern Med 2010;152:770-7.

4. Herman WH, Ma Y, Uwaifo G, et al. Differences in A1C by race and ethnicity among patients with impaired glucose tolerance in the Diabetes Prevention Program. Diabetes Care 2007;30:2453-7.

5. Chapp-Jumbo E, Edeoga C, Wan J, et al. Ethnic disparity in hemoglobin A1c levels among normoglycemic offspring of parents with type 2 diabetes mellitus. Endocr Pract 2012;18:356-62.

6. Haffner SM, D'Agostino R, Saad MF, et al. Increased insulin resistance and insulin secretion in nondiabetic African-Americans and Hispanics compared with non-Hispanic whites. The Insulin Resistance Atherosclerosis Study. Diabetes 1996;45:742-8.

7. Goldstein DE, Little RR, Lorenz RA, et al., American Diabetes Association. Tests of glycemia in diabetes. Diabetes Care 2003;26 (Suppl 1):S106-8.

8. Stettler C, Stahl M, Allemann S, et al. Association of 1,5-anhydroglucitol and 2-h postprandial blood glucose in type 2 diabetic patients. Diabetes Care 2008;31:1534-5.

9. Selvin E, Steffes MW, Ballantyne CM, et al. Racial differences in glycemic markers: a cross-sectional analysis of community-based data. Ann Intern Med 2011;154:303-9.

10. Herman WH, Dungan KM, Wolffenbuttel BH, et al. Racial and ethnic differences in mean plasma glucose, hemoglobin A1c, and 1,5-anhydroglucitol in over 2000 patients with type 2 diabetes. $J$ Clin Endocrinol Metab 2009:94:1689-94.

11. Friedman GD, Cutter GR, Donahue RP, et al. CARDIA: study design, recruitment, and some characteristics of the examined subjects. J Clin Epidemiol 1988;41:1105-16.
12. National Heart Lung and Blood Institute. The Coronary Artery Risk Development in Young Adults manual of operations: year 20-CARDIA VII exam 2005-2006. Birmingham, AL: CARDIA Coordinating Center, Division of Preventive Medicine, The University of Alabama at Birmingham. http://www.cardia.dopm.uab.edu/ exam-materials2/manual-of-operations/year-20 (accessed 10 Mar 2015).

13. Steffes M, Cleary P, Goldstein D, et al., the DCCT/EDIC Research Group. Hemoglobin A1c measurements over nearly two decades: sustaining comparable values throughout the Diabetes Control and Complications Trial and the Epidemiology of Diabetes Interventions and Complications Study. Clin Chem 2005;51:753-8.

14. Kohzuma T, Yamamoto T, Uematsu Y, et al. Basic performance of an enzymatic method for glycated albumin and reference range determination. J Diabetes Sci Technol 2011;5:1455-62.

15. Cefalu WT, Bell-Farrow AD, Petty M, et al. Clinical validation of a second-generation fructosamine assay. Clin Chem 1991;37:1252-6.

16. McGill JB, Cole TG, Nowatzke W, et al. Circulating 1,5-anhydroglucitol levels in adult patients with diabetes reflect longitudinal changes of glycemia: a U.S. trial of the GlycoMark assay. Diabetes Care 2004;27:1859-65.

17. Nowatzke W, Sarno MJ, Birch NC, et al. Evaluation of an assay for serum 1,5-anhydroglucitol (GlycoMark) and determination of reference intervals on the Hitachi 917 analyzer. Clin Chim Acta 2004;350:201-9.

18. Nathan DM, Steffes MW, Sun W, et al. Determining stability of stored samples retrospectively: the validation of glycated albumin. Clin Chem 2011;57:286-90.

19. Friedewald WT, Levy RI, Fredrickson DS. Estimation of the concentration of low-density lipoprotein cholesterol in plasma, without use of the preparative ultracentrifuge. Clin Chem 1972;18:499-502.

20. Little RR. Usefulness of glycated albumin assay for diabetes monitoring. J Diabetes Sci Technol 2011;5:1463-5.

21. Ford ES, Li C, Little RR, et al. Trends in A1C concentrations among U.S. adults with diagnosed diabetes from 1999 to 2004. Diabetes Care 2008;31:102-4.

22. Selvin E, Rawlings AM, Grams M, et al. Fructosamine and glycated albumin for risk stratification and prediction of incident diabetes and microvascular complications: a prospective cohort analysis of the Atherosclerosis Risk in Communities (ARIC) study. Lancet Diabetes Endocrinol 2014;2:279-88.

23. Juraschek SP, Steffes MW, Selvin E. Associations of alternative markers of glycemia with hemoglobin $\mathrm{A}(1 \mathrm{c})$ and fasting glucose. Clin Chem 2012;58:1648-55.

24. Sarwar N, Aspelund T, Eiriksdottir G, et al. Markers of dysglycaemia and risk of coronary heart disease in people without diabetes: Reykjavik prospective study and systematic review. PLoS Med 2010;7:e1000278.

25. Sarwar N, Gao P, Seshasai SR, et al. Diabetes mellitus, fasting blood glucose concentration, and risk of vascular disease: a collaborative meta-analysis of 102 prospective studies. Lancet 2010;375:2215-22.

26. Selvin E, Steffes MW, Zhu H, et al. Glycated hemoglobin, diabetes, and cardiovascular risk in nondiabetic adults. $N$ Engl $J$ Med 2010;362:800-11

27. Selvin E, Francis LMA, Ballantyne CM, et al. Nontraditional markers of glycemia. Diabetes Care 2011;34:960-7.

28. Selvin E, Rawlings AM, Grams M, et al. Association of 1,5-anhydroglucitol with diabetes and microvascular conditions. Clin Chem 2014;60:1409-18.

29. Juraschek SP, Steffes MW, Miller ER III, et al. Alternative markers of hyperglycemia and risk of diabetes. Diabetes Care 2012;35:2265-70.

30. Zaccardi F, Kurl S, Pitocco D, et al. Serum fructosamine and risk of type 2 diabetes mellitus among middle-age Finnish men: a 23-year population-based prospective study. Acta Diabetol 2015;52:161-6.

31. Malmstrom $\mathrm{H}$, Walldius $\mathrm{G}$, Grill V, et al. Fructosamine is a risk factor for myocardial infarction and all-cause mortality-longitudinal experience from the AMORIS cohort. Nutr Metab Cardiovasc Dis 2015;25:943-50.

32. Selvin $\mathrm{E}$, Rawlings $\mathrm{AM}$, Lutsey $\mathrm{PL}$, et al. Fructosamine and glycated albumin and the risk of cardiovascular outcomes and death. Circulation 2015;132:269-77.

33. Selvin E, Rawlings A, Lutsey $\mathrm{P}$, et al. Association of 1,5 -anhydroglucitol with cardiovascular disease and mortality. Diabetes 2016;65:201-8. 